\title{
Optimized simultaneous Multi-Frequency Lock-in Thermography
}

\author{
by M. Ricci*, S. Laureti*, M.K. Rizwan** and P. Burrascano** \\ * University of Calabria, Department of Informatics, Modeling, Electronics and System Engineering, Via P. Bucci \\ I-87036, Rende (CS), Italy, \{marco.ricci, stefano.laureti\}@unical.it \\ ** University of Perugia, Department of Engineering, Polo Scientifico Didattico di Terni, Strada di Pentima 4, \\ 05100, Terni (TR), Italy, \{muhammadkhalid.rizwan,pietro.burrascano\}@unipg.it
}

\begin{abstract}
An optimised simultaneous multi-frequency approach is here introduced for the first time as a completely novel active thermography scheme. A low peak-factor multi-frequency signal is designed and employed here for modulating the heat emission, being here a $400 \mathrm{~W}$ LED chips system in the visible spectral range. The analysis of the acquired thermograms in the frequency domain shows frequency-selective sensitivity at different depths within a carbon fiber reinforced composite sample containing delamination, meaning that multi-frequency lock-in can be performed at a set of desired frequencies with only a single measurement.
\end{abstract}

\section{Introduction}

In Active Thermography, as in all NDT techniques, much efforts are continuously devoted to maximizing the amount of information that can be inferred from a given test and to increase SNR.

In this framework, Pulsed Thermography (PT) [1] and Lock-In Thermography (LI) [2] represent the standard way to get the highest info - PT - or the maximum SNR - LI - respectively. However, since it is worth combining the advantages of both methods, in the last decades various waveforms for modulating the heat source emission such as Multi-Frequency Lock-in (MFLI) [3], Thermal Wave Radar or Pulse-compression Thermography (PuCT) were proposed [4], and postprocessing algorithms were developed, such as for instance Pulsed Phase Thermography (PPT) [5]

Among the various strategies that try to contextually optimize information' amount and SNR, the present paper focuses on MFLI. MFLI could represent a good tradeoff between SNR optimization and information amount since it relies on performing $\mathrm{LI}$ at a discrete set of various frequencies/thermal diffusion lengths. However, up to now, such method has been implemented in a non-optimal way.

In most of the cases, MFLI is nothing but a sequence of single-frequency LI tests done at different frequencies. Although in this case the frequencies can be selected arbitrarily, e.g. to have linearly spaced thermal diffusion lengths, the measurement procedure is not optimized and the measurement time is significantly increased, which is a serious drawback in the case of on-situ or in-line measurements.

To overcome this issue, some authors proposed the use of a square wave or other periodic excitations in order to simultaneously excite a set of discrete frequencies, whose amplitudes are dictated by the Fourier series representation of the periodic signal used [3]. Even if in this case the measurement time is reduced, the control on the spectral properties of the signals is limited.

Therefore, a nice breakthrough would be being able to design a modulating signal in which the number of tones as well their amplitudes would be maximally flexible, while optimizing the SNR for each inspection tone. To accomplish this goal, the theory of optimal synthesis of low peak-factor multi-tone signals can be very useful [6], and this concept is here introduced for the first time in the Active Thermography field.

To understand the concept of low-peak factor signals, let's introduce a generic multi-tone signal $s_{M}(t)$ as a combination of some sinusoidal tones that excites frequency components of interest with prescribed amplitudes and phase, as given in Eq.(1):

$$
s_{M}(t)=\sum_{k=1}^{N s} s_{k} * \sin \left(2 * p i * f_{k} * t+\phi_{k}\right)
$$

In Eq.(1), $s_{k}$ is the amplitude of the $k$-th sinusoid, $f_{k}$ and $\phi_{k}$ are the frequency and the phase of the $k$-th sinusoid respectively, and $N_{s}$ is the number of considered tones.

Assume also for simplicity that all $\left\{f_{k}\right\}$ are multiple of some fundamental frequency $f_{0}$, which can be not present in the summation. $f_{0}$ can be small enough so that the constrain on $\left\{f_{k}\right\}$ do not severely limit the choice of the tones' frequency. With this assumption, for a given choice of the $\left\{s_{k}\right\}$ and $\left\{f_{k}\right\}$ values, the shape of $s_{M}(t)$ depends on the values of the phase angles $\left\{\phi_{k}\right\}$, and it can be very different, even if the energy of the signal depends only on the $\left\{s_{k}\right\}$ values. Therefore, by considering that in practice the peak power of the excitation is limited, e.g. the max power of a heating/lighting system, it is preferable to choose $\left\{\phi_{k}\right\}$ such that the resulting peak factor of $s_{M}(t)$ is minimized, where the peak factor of a signal is the ratio between its peak-to-peak value and its root mean square value - a minimum peak factor guarantee the best SNR among the possible values of the phase angles $\left\{\phi_{k}\right\}$. It is known in literature that, if the tones are all multiple of a common fundamental frequency, then an expression for the phases $\left\{\phi_{k}\right\}$ exists that minimize the peak-factor even in presence of 
tones with different amplitudes $\left\{\mathbf{s}_{k}\right\}[6,7]$. Moreover, if the tones are equally spaced and have equal amplitudes, the resulting excitation exhibits a quite constant envelope and the lowest possible peak factor if:

$$
\phi_{\mathrm{k}}=-\pi * \frac{\mathrm{k}(\mathrm{k}-1)}{\mathrm{N}_{\mathrm{s}}}
$$

This means that different thermal waves having a discrete set of thermal wavelengths can be onset within the sample under test by using $s_{M}(t)$ as a modulation for the heat source emission.

An example of multi-tone input and output signals is reported in Fig.1, where $\left\{s_{k}\right\}$ range from $f_{1}=0.02 \mathrm{~Hz}$ up to $f_{38}=0.39 \mathrm{~Hz}$ and the fundamental frequency is $f_{0}=0.01 \mathrm{~Hz}$. The signal period is $T=1 / f_{0}$ and two periods are represented, which is turn used to modulate the emission of a $400 \mathrm{~W}$ LED system in the visible range. The sample tested is a CFRP sample with twelve plies and 9 simulated delaminations of area $20 \mathrm{~mm} \times 20 \mathrm{~mm}$ realized with Teflon tape at different depths from the inspected surface.

(a)

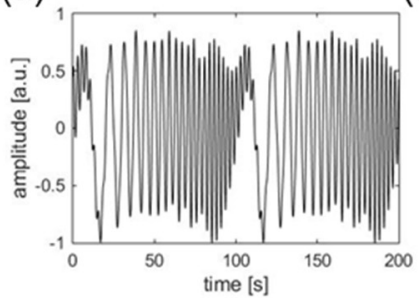

(b)

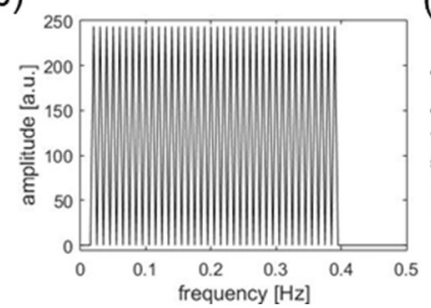

(c)

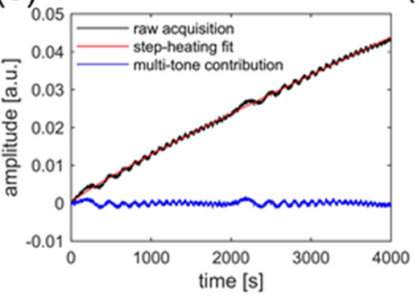

(d)

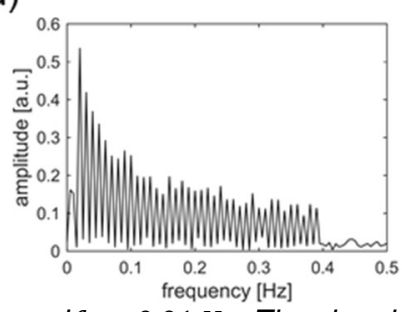

Fig. 1. (a), $s_{M}(t)$ multi-tone signal for $\left\{s_{k}\right\}$ ranging from $f_{1}=0.02 \mathrm{~Hz}$ up to $f_{38}=0.39 \mathrm{~Hz}$ and $f_{0}=0.01 \mathrm{~Hz}$. The signal period is $T=1 / f_{0}$ and two periods are represented; (b) the discrete spectrum of $s_{M}(t)$; (c) emissivity trend (black-solid line) of a single-pixel of a thermal image sequence collected by using $s_{M}(t)$ of subplot (a) for modulating a 8-LED heat source. After removing the step-heating contribute with a suitable fitting function (red-solid line), the pixel response to the sole multi-tone signal is obtained (blue-solid line). (d) the spectrum of the multi-tone output contribution represented in Fig.1-(c)

By applying the FFT pixel-wise to the acquired thermograms sequence, for each pixel a spectrum similar to that reported in Fig.1-(d) is obtained. Then, for each of the tones of the $s_{M}(t)$, two images are obtained as in standard LI method, one imaging the absolute value of the spectrum, the other visualizing the phase.

As an example, Figure 2 shows the images obtained by considering the absolute value of the FFT at some of the $f_{k}$ frequencies. As expected, lower frequencies can detect deeper defects - the sensitivity at different depths within the sample under test is showed by imaging different yet higher $f_{k}$.
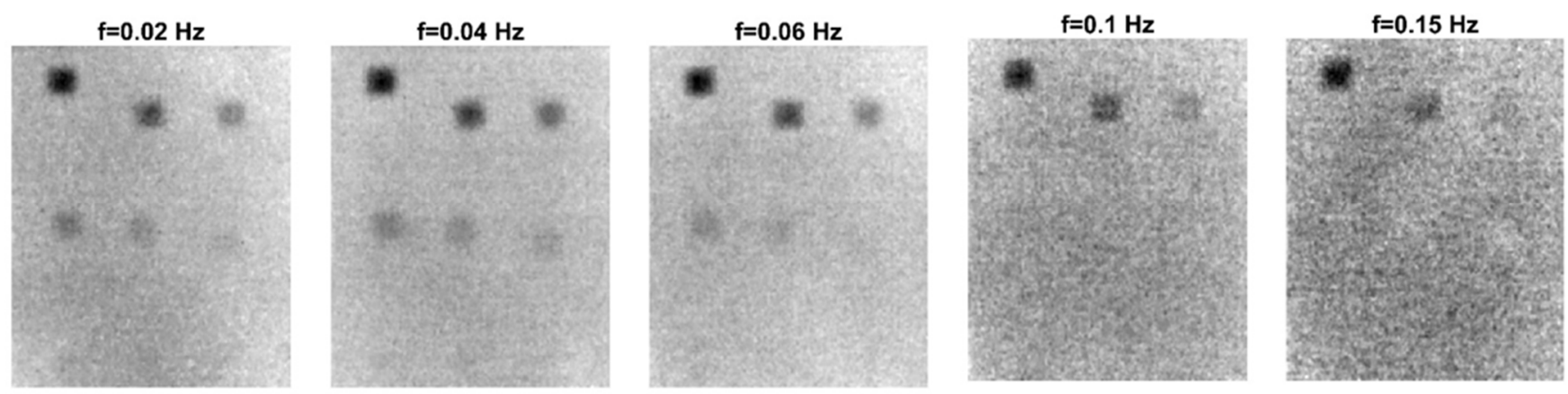

\section{Conclusions}

An optimised simultaneous multi-frequency approach is here introduced for the first time as a completely novel active thermography scheme. This is based on the combined use of a low peak-factor multi-frequency signal for modulating the heat source emission and the subsequent analysis of the acquired thermograms in the frequency domain. It is shown that the here-introduced scheme is a good candidate for implementing multi-frequency analysis with a single measurement the thermal responses of different depths within an artificially-delaminated CFRP sample are showed as a series of thermograms at different frequencies.

\section{REFERENCES}

[1] Maldague, X. (2000). Applications of infrared thermography in nondestructive evaluation. Trends in optical nondestructive testing, 591-609.

[2] Wu, D., \& Busse, G. (1998). Lock-in thermography for nondestructive evaluation of materials. Revue générale de thermique, 37(8), 693-703.

[3] Pitarresi, G. (2015). Lock-in signal post-processing techniques in infra-red thermography for materials structural evaluation. Experimental Mechanics, 55(4), 667-680. 
[4] Yi, Q., Malekmohammadi, H., Tian, G. Y., Laureti, S., \& Ricci, M. (2019). Quantitative Evaluation of Crack Depths on Thin Aluminum Plate using Eddy Current Pulse-Compression Thermography. IEEE Transactions on Industrial Informatics.

[5] Maldague, X., \& Marinetti, S. (1996). Pulse phase infrared thermography. Journal of applied physics, 79(5), 26942698.

[6] Schroeder, M. (1970). Synthesis of low-peak-factor signals and binary sequences with low autocorrelation (Corresp.). IEEE Transactions on Information Theory, 16(1), 85-89.

[7] Betta, G., Ferrigno, L., Laracca, M., Burrascano, P., Ricci, M., \& Silipigni, G. (2015). An experimental comparison of multi-frequency and chirp excitations for eddy current testing on thin defects. Measurement, 63, 207-220. 